Subscriber access provided by Caltech Library

\title{
Communication
}

\section{Electronic Modulation of Near-Field Radiative Transfer in Graphene Field Effect Heterostructures}

Nathan Thomas, Michelle C Sherrott, Jeremy J Brouillet, Harry A Atwater, and Austin J. Minnich

Nano Lett., Just Accepted Manuscript • DOI: 10.1021/acs.nanolett.9b01086 • Publication Date (Web): 29 May 2019

Downloaded from http://pubs.acs.org on May 30, 2019

\section{Just Accepted}

"Just Accepted" manuscripts have been peer-reviewed and accepted for publication. They are posted online prior to technical editing, formatting for publication and author proofing. The American Chemical Society provides "Just Accepted" as a service to the research community to expedite the dissemination of scientific material as soon as possible after acceptance. "Just Accepted" manuscripts appear in full in PDF format accompanied by an HTML abstract. "Just Accepted" manuscripts have been fully peer reviewed, but should not be considered the official version of record. They are citable by the Digital Object Identifier (DOI®). "Just Accepted" is an optional service offered to authors. Therefore, the "Just Accepted" Web site may not include all articles that will be published in the journal. After a manuscript is technically edited and formatted, it will be removed from the "Just Accepted" Web site and published as an ASAP article. Note that technical editing may introduce minor changes to the manuscript text and/or graphics which could affect content, and all legal disclaimers and ethical guidelines that apply to the journal pertain. ACS cannot be held responsible for errors or consequences arising from the use of information contained in these "Just Accepted" manuscripts. 


\title{
Electronic Modulation of Near-Field Radiative Transfer in Graphene Field Effect
}

\section{Heterostructures}

\author{
Nathan H. Thomas, ${ }^{\dagger}$ Michelle C. Sherrott,,$\uparrow$ Jeremy Broulliet, ${ }^{\ddagger}$ Harry A. \\ Atwater, ${ }^{\dagger, \ddagger}$ and Austin J. Minnich*,† \\ $\dagger$ Division of Engineering and Applied Science, California Institute of Technology, \\ Pasadena, California 91125, United States \\ $\ddagger$ Thomas J. Watson Laboratory of Applied Physics \\ ฯ Current address: Research Laboratory of Electronics, Massachusetts Institute of \\ Technology, Cambridge, MA, 02139 \\ E-mail: aminnich@caltech.edu
}

\begin{abstract}
Manipulating heat flow in a controllable and reversible manner is a topic of fundamental and practical interest. Numerous approaches to perform thermal switching have been reported, but they typically suffer from various limitations, for instance requiring mechanical modulation of a sub-micron gap spacing or only operating in a narrow temperature window. Here, we report the experimental modulation of radiative heat flow by electronic gating of a graphene field effect heterostructure without any moving elements. We measure a maximum heat flux modulation of $4 \pm 3 \%$ and an absolute modulation depth of $24 \pm 7 \mathrm{mWm}^{-2} \mathrm{~V}^{-1}$ in samples with vacuum gap distances ranging from 1 to 3 microns. The active area in the samples through which


heat is transferred is $\sim 1 \mathrm{~cm}^{2}$, indicating the scalable nature of these structures. A clear experimental path exists to realize switching ratios as large as $100 \%$, laying the foundation for electronic control of near-field thermal radiation using 2D materials.

Thermal switches that change thermal resistance in response to external stimuli have long been desired for temperature control applications. ${ }^{1}$ Typical thermal switches used in practice operate by mechanical mechanisms such as changing the physical contact of metallic leads, which are prone to failure. ${ }^{2}$ Numerous other schemes for controlling conductive heat flow have been proposed, including biasing of ferroelectrics, ${ }^{3-5}$ exploiting changes in properties across a phase transition, ${ }^{6-9}$ and magnetically aligning crystal networks. ${ }^{10}$ Thermal switches for radiative heat transfer can be realized if the optical dielectric constant or optical resonances at a surface can be altered by an external stimulus. ${ }^{11-14}$ In particular, theoretical and experimental works have described radiative heat flux modulation based on the insulator-metal transition of $\mathrm{VO}_{2} \cdot{ }^{15-18}$

Two-dimensional materials offer the capability to alter the surface dielectric function by electronic tuning of the free carrier concentration in a fast, controllable, and reversible manner without restrictions in operating temperature. ${ }^{19-25}$ Despite this capability, achieving even modest modulation of far-field radiative flux is challenging as thermal radiation is broadband. In the near-field, however, thermal radiation is primarily due to resonant coupling of narrowband surface modes, such as plasmons or phonon-polaritons. ${ }^{26,27}$ Additionally, nearfield radiative flux can be orders of magnitude larger than the far-field blackbody limit, and thus near-field radiative heat transport has been an area of intense experimental ${ }^{28-37}$ and theoretical ${ }^{38-46}$ interest.

Graphene has been proposed as an ideal material for thermal switching of near-field radiation as it exhibits a plasmonic resonance in the mid-infrared that can be electronically modulated. ${ }^{47-51}$ Recent experimental works have reported that graphene enables enhanced radiative thermal coupling between polar materials in the near-field. ${ }^{52,53}$ However, these studies do not take advantage of the electronic tunability of graphene to modulate heat 
flow. Recently, a theoretical scheme was proposed to electronically control radiative flow in a graphene field effect device, but an experimental implementation of a radiative thermal switch based on such a device has not yet been reported. ${ }^{54}$

We implemented an experiment to demonstrate such a thermal switch using a graphene heterostructure device. As shown schematically in Figure 1a, the top of the device is a graphene-coated silica optical flat, and the bottom is a back-gated, graphene-coated silicon wafer with a gate dielectric of $285 \mathrm{~nm}$ of $\mathrm{SiO}_{2}$ and $8 \mathrm{~nm}$ of $\mathrm{Al}_{2} \mathrm{O}_{3}$. An image of the sample on the experimental stage is shown in Figure 1b. The spring-loaded resistive heater presses the optical flat to the silicon wafer, which rests on a copper heat spreader with an embedded heat flux sensor (See Methods and SI Sec. 1).

We apply a standard fluctuational electrodynamics formalism ${ }^{55-57}$ to assess the potential for this sample configuration to modulate near-field radiative flow. In Figure 1c, we present calculations of the spectral thermal conductance, defined as $h(\omega)=\partial Q(\omega) / \partial T$, where $Q$ is the radiative heat flux, for our device at $T=300 K$ at various gap distances and Fermi levels. A clear change in spectral heat flux with Fermi level is evident. At vacuum gaps up to around $500 \mathrm{~nm}$, the hybrid phonon-plasmon-polariton can be tuned with the graphene Fermi level. ${ }^{54,58,59}$ However, this mode is tightly confined to the surface and does not contribute to heat flow at gap distances exceeding $1 \mu \mathrm{m}$. As a result, in the present experiments, where the vacuum separation distances between the top and bottom exceed 1 micron, the heat flux modulation originates only from the non-hybridized graphene plasmon. The spectral features from the $\mathrm{SiO}_{2}$ only contribute to the background heat flux. ${ }^{54}$ We obtain the total thermal conductance $h$ by integrating over all frequencies and define a thermal switching figure of merit as the ratio $h(\mu=0.3 \mathrm{eV}) / h(\mu=0.05 \mathrm{eV})$. This ratio is 2.1 at $d=100 \mathrm{~nm}$ for a total heat flux change over $100 \%$. At $d=1 \mu m$ the ratio is 1.1 , indicating a strong vacuum gap dependence on the influence of external bias to heat flux. Although at gap distances exceeding $1 \mu \mathrm{m}$ the optically active dielectric and substrate decrease the switching ratio, modulation should be observable with this experimentally achievable configuration. 

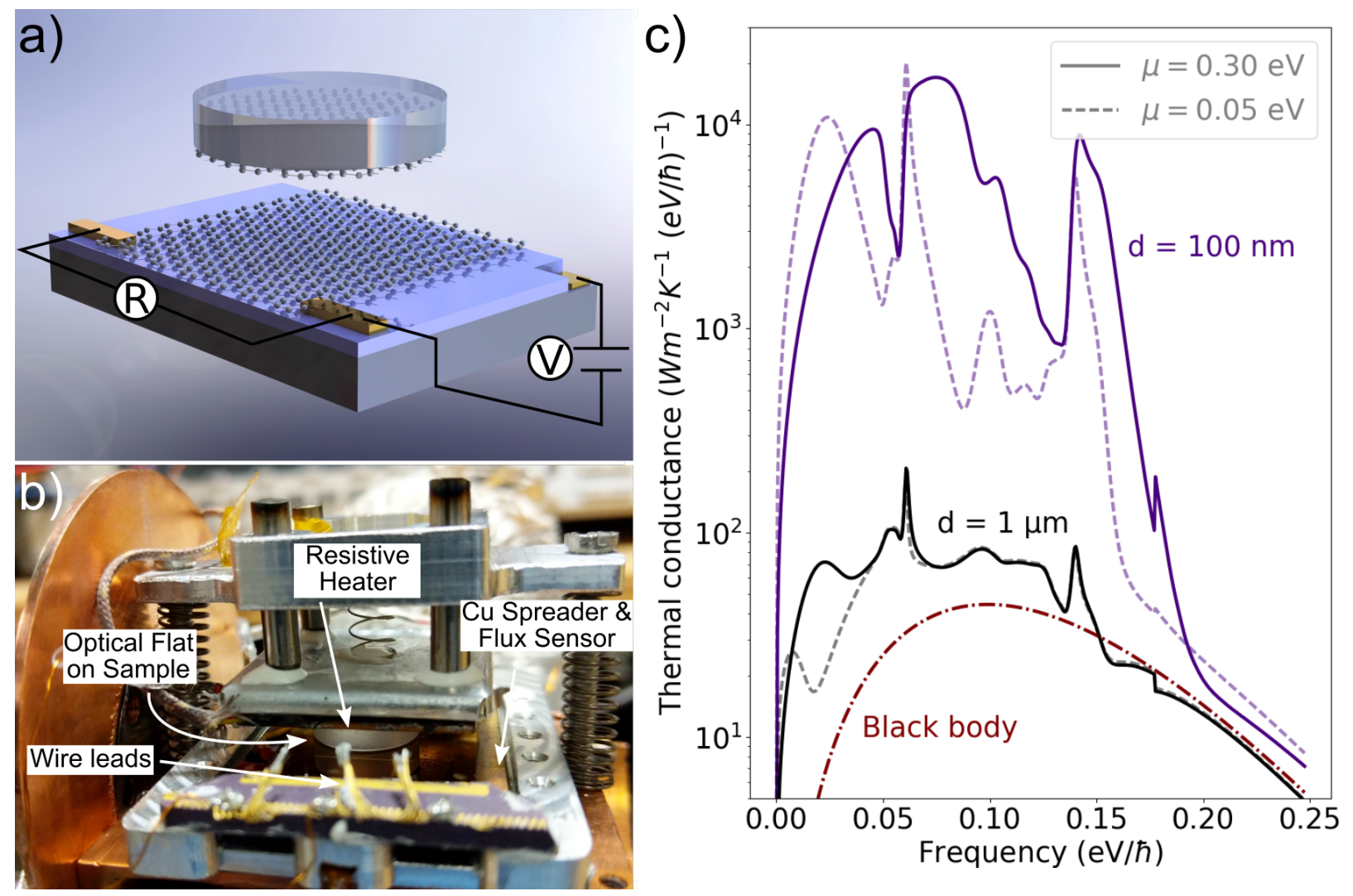

Figure 1: Experimental approach to measure near-field heat flux modulation. a, Schematic of sample configuration. The top of the heterostructure is an optical flat, coated with graphene. The bottom is a graphene-coated silicon wafer (grey) with $285 \mathrm{~nm}$ of thermally grown $\mathrm{SiO}_{2}$ and $8 \mathrm{~nm}$ of $\mathrm{ALD} \mathrm{Al}_{2} \mathrm{O}_{3}$ (purple). The graphene sheets are assumed to have the same Fermi level due to physical contact of the top and bottom. b, Image of the experimental stage with mounted sample. c, Calculated spectral thermal conductance at $300 \mathrm{~K}$ with a vacuum gap spacing of $100 \mathrm{~nm}$ (purple lines) and $1 \mu \mathrm{m}$ (black lines). The Fermi levels range from $0.05 \mathrm{eV}$ (dotted lines) to $0.3 \mathrm{eV}$ (solid lines), which are chosen as conservative estimates for the lower and upper magnitudes achievable for graphene with a charge neutral point near $0 \mathrm{~V}$ and a maximum applied voltage of $\pm 100 \mathrm{~V}$. The three sharp spectral features at frequencies above $0.06 \mathrm{eV}$ are due to the phonon-polariton resonances in the $\mathrm{SiO}_{2} \cdot{ }^{60}$ The broad feature at frequencies below $0.06 \mathrm{eV}$ originates from the graphene plasmon. 
The experiments are conducted in a cryostat cooled to $77 \mathrm{~K}$, where a resistive heater is used to heat the top of the sample. Once the temperatures equilibrate, the heat flux is monitored while a voltage ramp is applied to the back-gated bottom of the heterostructure via wire-bonded Au contacts (See Methods). Figures 2a-c show the measured heat flux versus time for three separate samples $\mathrm{S} 1, \mathrm{~S} 2$, and $\mathrm{S} 3$. The steady-state temperatures $T_{1}$ and $T_{2}$ for each sample are $197 \mathrm{~K}$ and $86 \mathrm{~K}$ for S1 and $270 \mathrm{~K}$ and $91 \mathrm{~K}$ for S2 and $269 \mathrm{~K}$ and $90 \mathrm{~K}$ for S3, respectively. For each sample, we observe a reversible change in the measured heat flux as the bias is ramped up and down. The magnitude of the modulation is around $0.5 \%$, $0.3 \%, 0.2 \%$, respectively. For samples S2 and S3, this effect is observed for multiple cycles. As a non-negligible thermal capacitance exists, there is a time delay from when the bias is applied and when the heat flux change is observed. For each sample, this delay was about 3 minutes. All samples were first ramped down to $-100 \mathrm{~V}$, which resulted in an observable change in heat flux. For S2, the bias could be ramped twice before sample failure. For S3, three cycles were possible, and the third ramp cycle was to a positive bias of $+95 \mathrm{~V}$.

A similar magnitude of heat flux change is observed for positive biases as negative biases. This result is expected because the graphene is slightly p-type with the charge neutral point found to be slightly positive, between $+5 \mathrm{~V}$ and $+15 \mathrm{~V}$. As a negative bias is applied, the hole concentration increases, the Fermi level becomes more negative, and the radiative thermal conductance increases. Applying a positive bias of $+95 \mathrm{~V}$ versus a negative bias of $-100 \mathrm{~V}$ results in a Fermi level of $0.29 \mathrm{eV}$ instead of $-0.32 \mathrm{eV}$, which is not sufficiently different in magnitude for a measurable change in heat flux considering the noise in the measurement.

From these data, we compute the heat flux versus electronic bias, shown in in Figures 2d-f for samples S1-S3, respectively. In each case, a linear fit is applied and a modest slope is visible, with the largest modulation exhibited in $\mathrm{S} 3$ with a slope of $24 \pm 7 \mathrm{mWm}^{-2} \mathrm{~V}^{-1}$. 

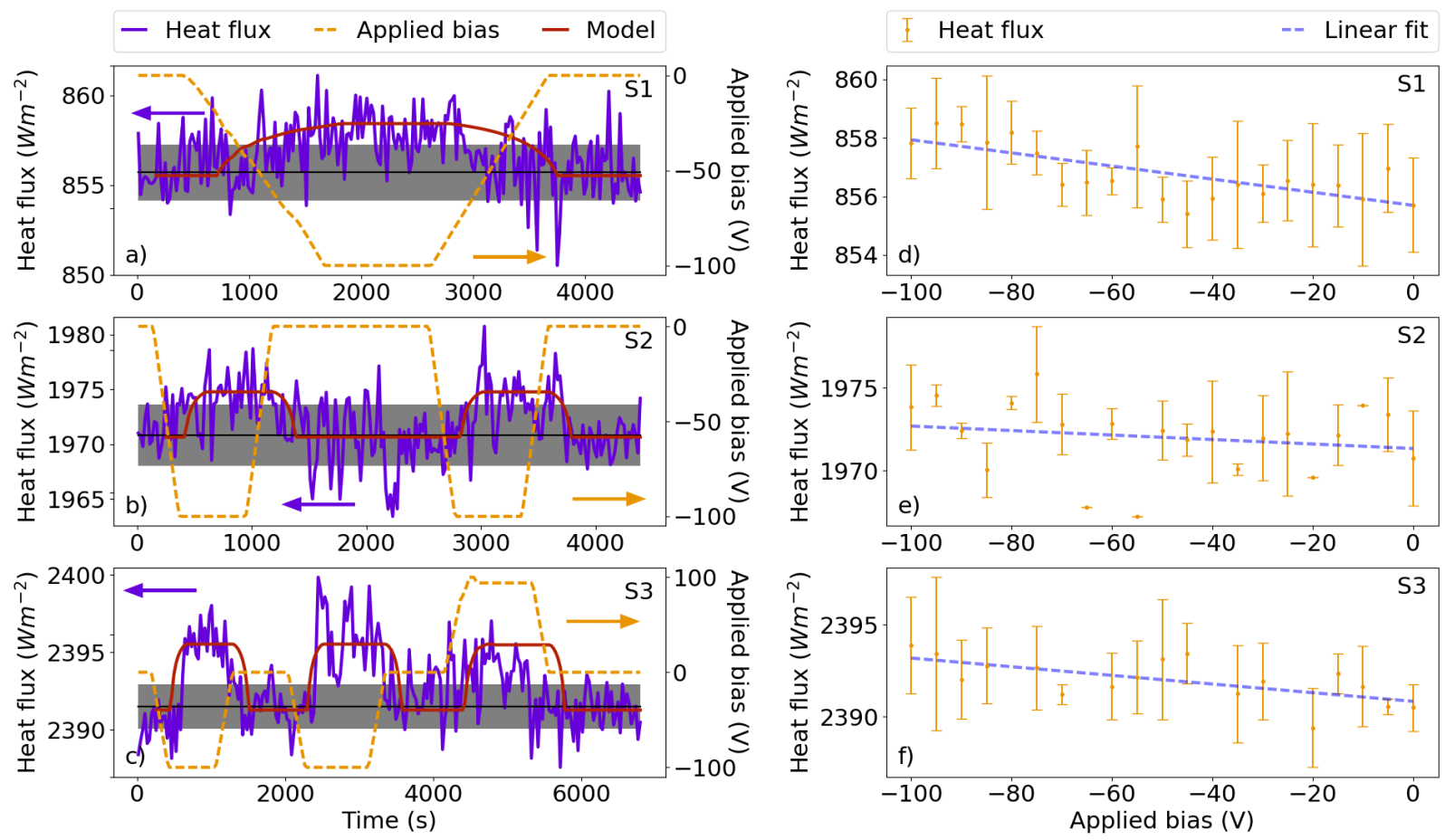

Figure 2: Heat flux modulation and modulation depth. a-c, Heat flux (purple), applied bias (orange dotted), and thermal model (red) versus time for three samples S1, S2, and S3, respectively. The black and grey lines indicate the mean and standard deviation of the signal at zero bias. d-f, Heat flux versus applied bias using data from Figures 2 a-c. The linear fit (blue dotted lines) indicate the greatest heat flux modulation occurs for S3, for which the modulation is $24 \pm 7 \mathrm{mWm}^{-2} \mathrm{~V}^{-1}$. 
We now examine the origin of this signal. First we rule out Joule heating by ensuring that that the leakage current does not exceed $350 \mathrm{nA}$ for these three samples, limiting parasitic Joule heating to a maximum of $0.26 \mathrm{Wm}^{-2}$. The observed heat flux change is on the order of $5 \mathrm{Wm}^{-2}$ in all cases, or nearly 20 times higher. For each measurement, we subtract the area normalized injected power $P=I V$. We also confirm independently that the injected Joule heat is not the cause of the modulated signal. For S3, the leakage power is approximately 20 times higher during the final voltage cycle than in the two previous cycles (see SI Sec. 6). If Joule heating were the source of the heat flux change, then in the final cycle the heat flux change would be $20 \times$ higher than in the previous cycles. As shown in Figure 2c, the change is nearly identical.

Next, we construct a thermal model. The heat flux measured in the experiment as a function of time consists of radiative flux and parasitic conductive losses:

$$
Q_{t o t}(t-\tau)=G\left(T_{1}-T_{2}\right)+Q_{r a d}\left(\mu(t), d, T_{1}, T_{2}\right)
$$

The first term accounts for the physical contact of the top and bottom surfaces with a conductance $G$. The second term, $Q_{\text {rad }}$, is the radiative heat flux between the planar surfaces. ${ }^{30,56,61}$ In the model, the temperatures are fixed and the radiative thermal conductance changes due to the changing Fermi level. The time dependence in the heat flux is accounted for in the time-dependent nature of the voltage ramp and phenomenologically in the adjustable time delay $\tau$ between the start of the voltage ramp and when the heat flux sensor registers the flux change. Since the top and bottom are likely in physical contact, it is assumed that the two graphene sheets are shorted such that $\mu_{1}=\mu_{2}=\mu$. The values for $\mu, T_{1}$, and $T_{2}$ are measured in the experiment (See Methods and SI Sec. 6). We use optical interferometric measurements to estimate the effective gap distance $d$, and separate measurements without spacers to estimate the value of the parasitic conductance $G$. The final values for $d$ and $G$ for each sample are obtained in two independent fitting procedures, using the measured heat 
flux change and the calculated radiative flux (see SI. Sec. 6).

The model predictions are plotted in Figures 2a-c and agree well with the measured heat flux. For samples S1-S3, the fitted values for $G$ are $6.6 \pm 0.2,8.9 \pm 0.2$, and $11.3 \pm$ $0.2 \mathrm{Wm}^{-2} \mathrm{~K}^{-1}$, respectively, and for $d$ are $2.5 \pm 0.2,2.3 \pm 0.2$, and $2.3 \pm 0.13$ microns, respectively. These values indicate non-negligible physical contact between the optical flat and the bottom substrate and also a vacuum gap, commensurate with previous near-field heat transfer experiments. ${ }^{28}$ After the initial time delay, the heat flux increases to its steadystate value and then decreases to the equilibrium value, following the measured heat flux. During the final, third voltage ramp for S3, there is a slight deviation from the model at later stages when the peak voltage of $+95 \mathrm{~V}$ was applied. We attribute this discrepancy to the sample dielectric beginning to break down, decreasing the electric field effect and subsequently lowering the Fermi level compared to that used in the model.

With the model and the fitted gap distances, we can also estimate the absolute radiative heat flux between the two surfaces. Subtracting the conductive contribution, we find the radiative heat flux for $\mathrm{S} 1$ is $125 \pm 9 \mathrm{Wm}^{-2}$, exceeding the black body limit of $84 \pm 4 \mathrm{Wm}^{-2}$ by $50 \pm 10 \%$. For samples $\mathrm{S} 2$ and $\mathrm{S} 3$, the thermal emitter is approximately $80 \mathrm{~K}$ hotter than for $\mathrm{S} 1$, and the radiative heat flux is $385 \pm 26 \mathrm{Wm}^{-2}$ and $381 \pm 18 \mathrm{Wm}^{-2}$ for $\mathrm{S} 2$ and S3, respectively. Sample S2 exceeds the black body limit of $299 \pm 9 \mathrm{Wm}^{-2}$ by $29 \pm 7 \%$, and sample S3 exceeds the black body limit of $295 \pm 9 \mathrm{Wm}^{-2}$ by $29 \pm 9 \%$. These results confirm that the radiative transport is in the near-field regime (see SI Sec. 7).

We next present the heat flux versus hot side temperature for sample S4 for two different biases, as shown in Figure 3a. The heat flux is first measured at zero bias and then at -35 V. Additional measurements at temperatures below the third point at $175 \mathrm{~K}$ were not possible due to dielectric breakdown. We again interpret these results using the model described previously, where here we fit for $G, d$, and $\mu$ (see SI Sec. 6). Using the fitting parameters from the zero bias data, we then apply a zero-parameter fit for the measurement under 

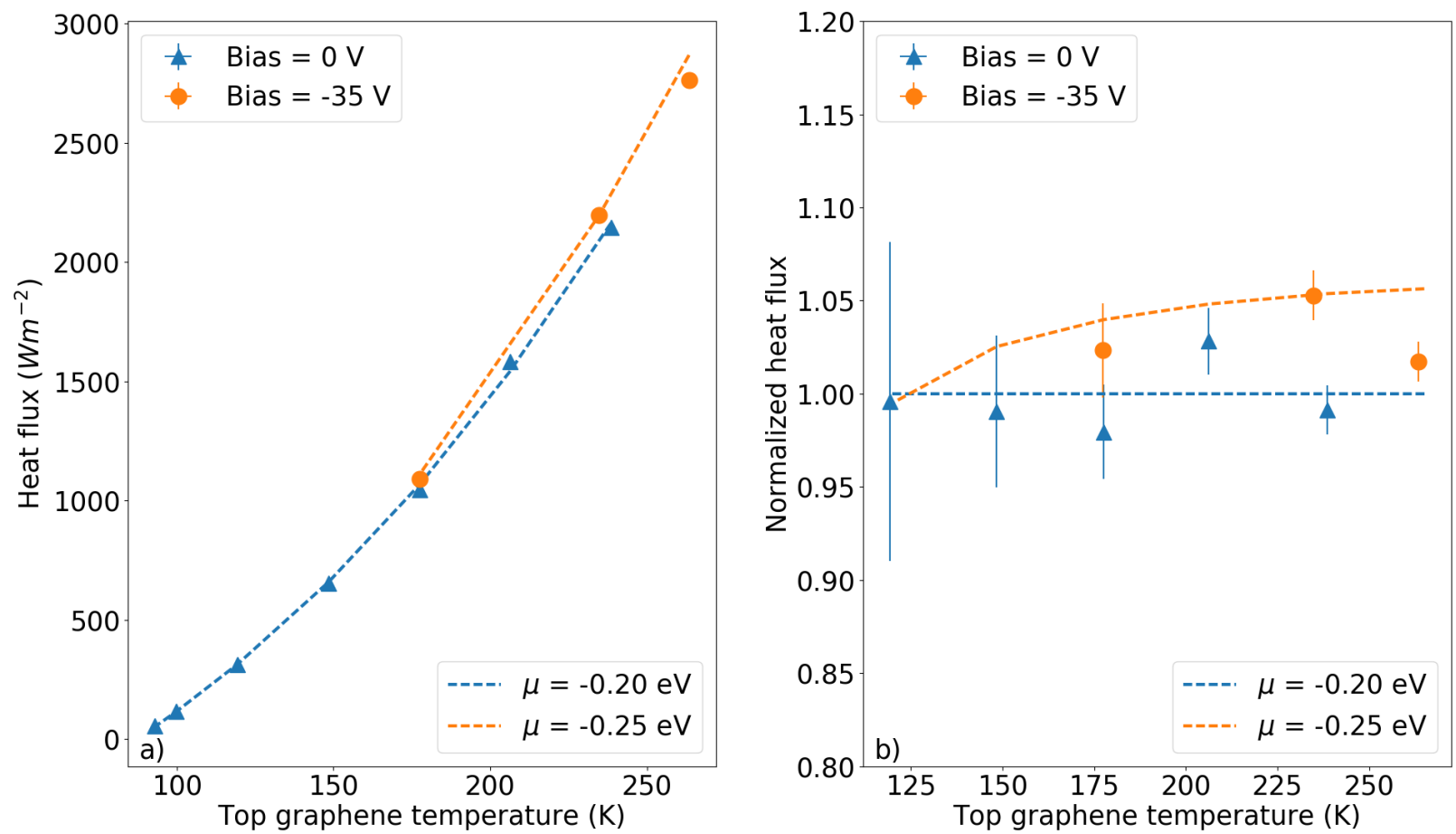

Figure 3: Heat flux versus temperature at different voltages. a, Measured heat flux versus temperature for sample $\mathrm{S} 4$ for $0 \mathrm{~V}$ (blue triangles) and $-35 \mathrm{~V}$ (orange circles). The blue dotted line is a fit of Eq. 1 to the blue data points, for which $d=560 \mathrm{~nm}, G=5.07$ $\mathrm{Wm}^{-2} \mathrm{~K}^{-1}$, and $\mu=-0.20 \mathrm{eV}$. The orange line is a zero parameter fit, using the these values and a Fermi level of $\mu=-0.25 \mathrm{eV}$ corresponding to the $-35 \mathrm{~V}$ bias. $\mathbf{b}$, Normalized heat flux versus temperature for the two biases. The heat flux under bias is greater than the zero bias case by around $3-5 \%$. 
bias, accounting for the change in Fermi level. The model shows good agreement with the measured heat flux.

In Figure 3b, the data are normalized to the dotted blue fit. Point to point variation in both signals is evident, but there is also a clear trend that heat flux in the biased case is greater than that in the zero bias case by around 3-5\%. At $175 \mathrm{~K}$, where both the biased and zero bias case were measured and a more direct comparison is possible, the heat flux change is $4 \pm 3 \%$.

Although the modulation reported here is of the order of a few percent, as expected due to the micron-scale gap spacing, improvements to the experimental setup should allow for modulation values approaching 100\%. Primarily, the gate dielectric must exhibit high breakdown strength approaching that of bulk $\mathrm{SiO}_{2}$ while also exhibiting warping less than $100 \mathrm{~nm}$ over the substrate area. These qualities would allow for larger biases, small vacuum gaps, and reduced conductive contact. By eliminating all conductive losses and reducing the gap spacing to $100 \mathrm{~nm}$, biasing to $-100 \mathrm{~V}$ as in these experiments would result in a heat flux modulation of $100 \%$. Reducing the gap distance to such a value would also allow for coupling between the graphene plasmon and the phonon-polariton in the dielectric to influence heat flow. ${ }^{54}$ However, even reducing the gap to $500 \mathrm{~nm}$ without any change in the interface conductance would lead to modulation of $45 \%$.

In summary, we report the experimental demonstration of the modulation of near-field thermal radiation by electronic gating of $1 \mathrm{~cm}^{2}$ scale graphene heterostructures. The maximum measured modulation was $4 \pm 3 \%$, and the maximum measured modulation depth was $24 \pm 7 \mathrm{mWm}^{-2} \mathrm{~V}^{-1}$. This work demonstrates that two-dimensional materials can be used to electronically control near-field radiative transfer and provides a path for realizing thermal switches with modulation depth approaching $100 \%$. 


\section{Methods}

Sample Fabrication. The bottom substrate is a silicon wafer with a thermally grown oxide of $285 \mathrm{~nm}$ thickness. On its own, the thermally grown $\mathrm{SiO}_{2}$ is not adequate for biasing to large voltages of $\sim 100 \mathrm{~V}$, and an extra $8 \mathrm{~nm}$ layer of $\mathrm{Al}_{2} \mathrm{O}_{3}$ was deposited using atomic layer deposition to increase breakdown strength. The wafers are cleaved to pieces $25.4 \mathrm{~mm} \times$ $31.75 \mathrm{~mm}$ and are cleaned in isopropyl alcohol in a sonicator, followed by an $\mathrm{O}_{2}$ plasma clean and an overnight soak in Piranha solution. Graphene pieces $15 \mathrm{~mm} \times 20 \mathrm{~mm}$ are transferred to the substrate by a modified wet transfer technique (see SI Sec. 2). The top substrate is an optical flat, $12.7 \mathrm{~mm}$ in diameter, which is cleaned and has graphene transferred to it in an identical manner. Gold bonding contacts and the array of $\mathrm{SiO}_{2}$ posts, each $1 \mu \mathrm{m}$ square and spaced $500 \mu \mathrm{m}$ apart, are fabricated on the bottom substrate by electron beam lithography and subsequent electron beam evaporation. Of the four different samples studied here, the pillars are grown to $200 \mathrm{~nm}$ tall for samples S1, S2, and S4, and $400 \mathrm{~nm}$ tall for sample S3.

Experimental Protocol. After fabrication, the optical flat is pressed down onto the bottom substrate by a spring loaded resistive heater, shown in Figure 1c. Beneath the sample is a thermopile heat flux sensor, which has been calibrated at temperatures from $90 \mathrm{~K}$ to $300 \mathrm{~K}$ by measuring the output signal as a function of input power into a resistive heater (see SI Sec. 3). A copper heat spreader is secured to the top of the sensor with thermally conductive epoxy. The entire array sits on a copper base that is screwed to the cold finger of a cryostat that is pumped down to $1 \times 10^{-6}$ Torr during the experiment. The external bias is applied through wire bonded gold pads on the sample. The temperatures of the cold finger and heater are measured with a Si-diode and a K-type thermocouple, respectively, and are maintained through two independent PID controllers. The copper base and the heat flux sensor temperatures are measured by K-type thermocouples, and the graphene surface resistance is recorded with a Fluke multi-meter while the sample is biased with a Keithley 2410 source meter.

The cold finger is cooled to $77 \mathrm{~K}$ with a liquid nitrogen feed and the resistive heater is 
set to a maximum input power. The equilibrium temperature of the heterostructure bottom reaches between $86 \mathrm{~K}$ and $91 \mathrm{~K}$, but the maximum temperature of the resistive heater is dependent on the nitrogen flow rate. For sample S1, the top temperature reaches $197 \mathrm{~K}$. For samples S2 and S3, the top temperature reaches $269 \mathrm{~K}$, and $270 \mathrm{~K}$, respectively. For the measurements of heat flux over time, after the heat flux sensor has equilibrated, the sample voltage is applied at a constant ramp rate. It was found that equilibration can take several hours, so there is a linear drift in the heat flux signal that is corrected (see SI Sec. 4). The ramp rate varies from sample to sample as the quality of the gate dielectric varies for each sample. The leakier the gate dielectric, the slower the ramp. The fastest ramp was $0.4 \mathrm{Vs}^{-1}$ for S2 and S3 and the slowest was $0.1 \mathrm{Vs}^{-1}$ for $\mathrm{S} 1$. After the voltage is held at its maximum value for around 15 minutes, the voltage is ramped down at the same rate.

The measurement of heat flux at different temperatures follows a similar procedure, although the two bias conditions are tracked separately. First, the heat flux was measured at different temperatures at zero bias, and then the heat flux was measured at different temperature at $-35 \mathrm{~V}$. Again, the maximum heater temperature is determined by the upper limit of the heater input power. Over the course of a measurement, the liquid nitrogen flow rate falls with the pressure within the dewar. The maximum temperature is higher for the biased case over the zero-bias case because the flow rate and therefore the cooling power of the liquid nitrogen to the cold finger was lower. For all samples tested here, we find that after a few ramping cycles the gate dielectric breaks down, limiting the amount of data that can be obtained for each sample.

Simulations and Modeling. A transfer matrix method is used to calculate the Fresnel coefficients $r_{1}$ and $r_{2}$ in the heat flux calculation for planar media, ${ }^{46}$ with the graphene modeled as a conducting surface with a local optical conductivity. ${ }^{62}$ See SI Sec. 9 for optical properties of $\mathrm{SiO}_{2}$ and $\mathrm{Al}_{2} \mathrm{O}_{3}$. The graphene conductivity was calculated at $300 \mathrm{~K}$ with a scattering time of $\tau=50 \times 10^{-15} \mathrm{~s}$, which reasonably corresponds to the quality of graphene films grown by chemical vapor deposition. ${ }^{63}$ 
Using the measured applied voltage, we apply a capacitor model to determine the Fermi levels. To determine the temperatures of the graphene surfaces, we employ a thermal resistor model using our knowledge of the heat flux and the measured temperatures of the thermal sinks (in this case, the heater and the copper heat spreader of the heat flux sensor, see SI. Sec. 1). ${ }^{36}$ To first approximation, we assume that the thermal resistance between top and bottom samples is much greater than that between the bottom sample and the copper heat spreader. As the bottom sample substrate is silicon, which has a comparatively high thermal conductivity, the bottom graphene surface temperature is approximately equal to that of the heat flux sensor. For the top sample, a considerable temperature drop occurs across the optical flat, and the resistor model is necessary (see SI Sec. 6).

\section{Acknowledgements}

This work is part of the Light-Material Interactions in Energy Conversion Energy Frontier Research Center funded by the U.S. Department of Energy, Office of Science, Office of Basic Energy Sciences under Award No. DE-SC0001293. This material is based upon work supported by the National Science Foundation Graduate Research Fellowship under Grant No. 1144469. MCS gratefully acknowledges fellowship support from the Resnick Sustainability Institute. The authors would like to recognize the Kavli NanoScience Institute at Caltech and to thank Dr. Ognjen Ilic for insightful conversation.

\section{Author contributions}

N.H.T and A.J.M conceived and designed the experiment. N.H.T fabricated samples and conducted numerical simulations. M.C.S assisted in design and sample fabrication. J.B assisted in sample fabrication. N.H.T, H.A.A, and A.J.M wrote the manuscript. All authors commented and approved the manuscript. 


\section{Supporting Information Available}

Sample schematic, graphene wet transfer technique, heat flux sensor calibration, background heat flux analysis, signal drift correction, fitting procedure for thermal model, signal to noise \& uncertainty, leakage current analysis, optical properties

\section{References}

(1) Wehmeyer, G.; Yabuki, T.; Monachon, C.; Wu, J.; Dames, C. Thermal diodes, regulators, and switches: Physical mechanisms and potential applications. Appl. Phys. Rev. 2017, 4, 041304.

(2) Shu, Q. S.; Demko, J. A.; Fesmire, J. E. Heat switch technology for cryogenic thermal management. IOP Conf. Ser.: Mater. Sci. Eng 2017, 278, 012133.

(3) Ihlefeld, J. F.; Foley, B. M.; Scrymgeour, D. A.; Michael, J. R.; McKenzie, B. B.; Medlin, D. L.; Wallace, M.; Trolier-McKinstry, S.; Hopkins, P. E. Room-Temperature Voltage Tunable Phonon Thermal Conductivity via Reconfigurable Interfaces in Ferroelectric Thin Films. Nano Lett. 2015, 15, 1791-1795.

(4) Foley, B. M.; Wallace, M.; Gaskins, J. T.; Paisley, E. A.; Johnson-Wilke, R. L.; Kim, J.W.; Ryan, P. J.; Trolier-McKinstry, S.; Hopkins, P. E.; Ihlefeld, J. F. Voltage-Controlled Bistable Thermal Conductivity in Suspended Ferroelectric Thin-Film Membranes. ACS Appl. Mater. Interfaces 2018, 10, 25493-25501.

(5) Seijas-Bellido, J. A.; Escorihuela-Sayalero, C.; Royo, M.; Ljungberg, M. P.; Wojde, J. C.; iguez, J.; Rurali, R. A phononic switch based on ferroelectric domain walls. Phys. Rev. B 2017, 96, 140101.

(6) Zheng, R.; Gao, J.; Wang, J.; Chen, G. Reversible temperature regulation of electrical 
and thermal conductivity using liquidsolid phase transitions. Nat. Commun. 2011, 2, 289.

(7) Choe, H. S.; Suh, J.; Ko, C.; Dong, K.; Lee, S.; Park, J.; Lee, Y.; Wang, K.; Wu, J. Enhancing Modulation of Thermal Conduction in Vanadium Dioxide Thin Film by Nanostructured Nanogaps. Sci. Rep. 2017, 7, 7131.

(8) Chen, R.; Cui, Y.; Tian, H.; Yao, R.; Liu, Z.; Shu, Y.; Li, C.; Yang, Y.; Ren, T.; Zhang, G.; Zou, R. Controllable Thermal Rectification Realized in Binary Phase Change Composites. Sci. Rep. 2015, 5, 8884 .

(9) Kim, K.; Kaviany, M. Thermal conductivity switch: Optimal semiconductor/metal melting transition. Phys. Rev. B 2016, 94, 155203.

(10) Shin, J.; Kang, M.; Tsai, T.; Leal, C.; Braun, P. V.; Cahill, D. G. Thermally Functional Liquid Crystal Networks by Magnetic Field Driven Molecular Orientation. ACS Macro Lett. 2016, 5, 955-960.

(11) Otey, C. R.; Lau, W. T.; Fan, S. Thermal Rectification through Vacuum. Phys. Rev. Lett. 2010, 104, 154301.

(12) Basu, S.; Francoeur, M. Near-field radiative transfer based thermal rectification using doped silicon. Appl. Phys. Lett. 2011, 98, 113106.

(13) Iizuka, H.; Fan, S. Rectification of evanescent heat transfer between dielectric-coated and uncoated silicon carbide plates. J. Appl. Phys. 2012, 112, 024304.

(14) Kou, J.; Minnich, A. J. Dynamic optical control of near-field radiative transfer. Opt. Express 2018, 26, A729-A736.

(15) Ben-Abdallah, P.; Biehs, S.-A. Near-Field Thermal Transistor. Phys. Rev. Lett. 2014, 112, 044301. 
(16) Ito, K.; Nishikawa, K.; Iizuka, H.; Toshiyoshi, H. Experimental investigation of radiative thermal rectifier using vanadium dioxide. Appl. Phys. Lett 2014, 105, 253503.

(17) Ito, K.; Nishikawa, K.; Miura, A.; Toshiyoshi, H.; Iizuka, H. Dynamic Modulation of Radiative Heat Transfer beyond the Blackbody Limit. Nano Lett. 2017, 17, 4347-4353.

(18) Fiorino, A.; Thompson, D.; Zhu, L.; Mittapally, R.; Biehs, S.-A.; Bezencenet, O.; ElBondry, N.; Bansropun, S.; Ben-Abdallah, P.; Meyhofer, E.; Reddy, P. A Thermal Diode Based on Nanoscale Thermal Radiation. ACS Nano 2018, 12, 5774-5779.

(19) Wang, F.; Zhang, Y.; Tian, C.; Girit, C.; Zettl, A.; Crommie, M.; Shen, Y. R. GateVariable Optical Transitions in Graphene. Science 2008, 320, 206-209.

(20) Freitag, M.; Chiu, H.-Y.; Steiner, M.; Perebeinos, V.; Avouris, P. Thermal infrared emission from biased graphene. Nat. Nanotechnol. 2010, 5, 497-501.

(21) Ju, L.; Geng, B.; Horng, J.; Girit, C.; Martin, M.; Hao, Z.; Bechtel, H. A.; Liang, X.; Zettl, A.; Shen, Y. R.; Wang, F. Graphene plasmonics for tunable terahertz metamaterials. Nat. Nanotechnol. 2011, 6, 630-634.

(22) Fang, Z.; Thongrattanasiri, S.; Schlather, A.; Liu, Z.; Ma, L.; Wang, Y.; Ajayan, P. M.; Nordlander, P.; Halas, N. J.; Garca de Abajo, F. J. Gated Tunability and Hybridization of Localized Plasmons in Nanostructured Graphene. ACS Nano 2013, 7, 2388-2395.

(23) Brar, V. W.; Sherrott, M. C.; Jang, M. S.; Kim, S.; Kim, L.; Choi, M.; Sweatlock, L. A.; Atwater, H. A. Electronic modulation of infrared radiation in graphene plasmonic resonators. Nat. Commun. 2015, 6, 7032.

(24) Sherrott, M. C.; Hon, P. W. C.; Fountaine, K. T.; Garcia, J. C.; Ponti, S. M.; Brar, V. W.; Sweatlock, L. A.; Atwater, H. A. Experimental Demonstration of >230 Phase Modulation in Gate-Tunable GrapheneGold Reconfigurable Mid-Infrared Metasurfaces. Nano Lett. 2017, 17, 3027-3034. 
(25) Lee, J.; Wang, Z.; He, K.; Yang, R.; Shan, J.; Feng, P. X.-L. Electrically tunable singleand few-layer MoS2 nanoelectromechanical systems with broad dynamic range. Sci. Adv. 2018, 4, eaao6653.

(26) Shchegrov, A. V.; Joulain, K.; Carminati, R.; Greffet, J.-J. Near-Field Spectral Effects due to Electromagnetic Surface Excitations. Phys. Rev. Lett. 2000, 85, 1548-1551.

(27) Song, B.; Fiorino, A.; Meyhofer, E.; Reddy, P. Near-field radiative thermal transport: From theory to experiment. AIP Adv. 2015, 5, 053503.

(28) Hu, L.; Narayanaswamy, A.; Chen, X.; Chen, G. Near-field thermal radiation between two closely spaced glass plates exceeding Plancks blackbody radiation law. Appl. Phys. Lett. 2008, 92, 133106.

(29) Shen, S.; Narayanaswamy, A.; Chen, G. Surface Phonon Polaritons Mediated Energy Transfer between Nanoscale Gaps. Nano Lett. 2009, 9, 2909-2913.

(30) Narayanaswamy, A.; Shen, S.; Chen, G. Near-field radiative heat transfer between a sphere and a substrate. Phys. Rev. B 2008, 78, 115303.

(31) Lang, S.; Sharma, G.; Molesky, S.; Krnzien, P. U.; Jalas, T.; Jacob, Z.; Petrov, A. Y.; Eich, M. Dynamic measurement of near-field radiative heat transfer. Sci. Rep. 2017, 7, 13916.

(32) Song, B.; Thompson, D.; Fiorino, A.; Ganjeh, Y.; Reddy, P.; Meyhofer, E. Radiative heat conductances between dielectric and metallic parallel plates with nanoscale gaps. Nat. Nanotechnol. 2016, 11, 509-514.

(33) Watjen, J. I.; Zhao, B.; Zhang, Z. M. Near-field radiative heat transfer between dopedSi parallel plates separated by a spacing down to 200nm. Appl. Phys. Lett. 2016, 109, 203112 . 
(34) Kim, K.; Song, B.; Fernndez-Hurtado, V.; Lee, W.; Jeong, W.; Cui, L.; Thompson, D.; Feist, J.; Reid, M. T. H.; Garca-Vidal, F. J.; Cuevas, J. C.; Meyhofer, E.; Reddy, P. Radiative heat transfer in the extreme near field. Nature 2015, 528, 387-391.

(35) Bernardi, M. P.; Milovich, D.; Francoeur, M. Radiative heat transfer exceeding the blackbody limit between macroscale planar surfaces separated by a nanosize vacuum gap. Nat. Commun. 2016, 7, 12900.

(36) Ito, K.; Miura, A.; Iizuka, H.; Toshiyoshi, H. Parallel-plate submicron gap formed by micromachined low-density pillars for near-field radiative heat transfer. Appl. Phys. Lett. 2015, 106, 083504.

(37) Song, B.; Ganjeh, Y.; Sadat, S.; Thompson, D.; Fiorino, A.; Fernndez-Hurtado, V.; Feist, J.; Garcia-Vidal, F. J.; Cuevas, J. C.; Reddy, P.; Meyhofer, E. Enhancement of near-field radiative heat transfer using polar dielectric thin films. Nat. Nanotechnol. 2015, 10, 253-258.

(38) Zhang, Z. M.; Basu, S. Entropy flow and generation in radiative transfer between surfaces. Int. J. Heat Mass Transfer 2007, 50, 702-712.

(39) Guo, Y.; Jacob, Z. Thermal hyperbolic metamaterials. Opt. Express 2013, 21, 1501415019.

(40) Rodriguez, A. W.; Ilic, O.; Bermel, P.; Celanovic, I.; Joannopoulos, J. D.; Soljai, M.; Johnson, S. G. Frequency-Selective Near-Field Radiative Heat Transfer between Photonic Crystal Slabs: A Computational Approach for Arbitrary Geometries and Materials. Phys. Rev. Lett. 2011, 107, 114302.

(41) Basu, S.; Wang, L. Near-field radiative heat transfer between doped silicon nanowire arrays. Appl. Phys. Lett. 2013, 102, 053101. 
(42) Joulain, K.; Drevillon, J.; Ben-Abdallah, P. Noncontact heat transfer between two metamaterials. Phys. Rev. B 2010, 81, 165119.

(43) Chen, K.; Santhanam, P.; Sandhu, S.; Zhu, L.; Fan, S. Heat-flux control and solid-state cooling by regulating chemical potential of photons in near-field electromagnetic heat transfer. Phys. Rev. B 2015, 91, 134301.

(44) Ding, D.; Kim, T.; Minnich, A. J. Active thermal extraction of near-field thermal radiation. Phys. Rev. B 2016, 93, 081402.

(45) Chiloyan, V.; Garg, J.; Esfarjani, K.; Chen, G. Transition from near-field thermal radiation to phonon heat conduction at sub-nanometre gaps. Nat. Commun. 2015, 6, 6755 .

(46) Narayanaswamy, A.; Zheng, Y. A Green's function formalism of energy and momentum transfer in fluctuational electrodynamics. J. Quant. Spectrosc. Radiat. Transfer 2014, 132, $12-21$.

(47) Ilic, O.; Jablan, M.; Joannopoulos, J. D.; Celanovic, I.; Buljan, H.; Soljai, M. Near-field thermal radiation transfer controlled by plasmons in graphene. Phys. Rev. B 2012, 85, 155422 .

(48) Svetovoy, V. B.; van Zwol, P. J.; Chevrier, J. Plasmon enhanced near-field radiative heat transfer for graphene covered dielectrics. Phys. Rev. B 2012, 85, 155418.

(49) Ben-Abdallah, P.; Belarouci, A.; Frechette, L.; Biehs, S.-A. Heat flux splitter for nearfield thermal radiation. Appl. Phys. Lett. 2015, 10\%, 053109.

(50) Ilic, O.; Thomas, N. H.; Christensen, T.; Sherrott, M. C.; Soljai, M.; Minnich, A. J.; Miller, O. D.; Atwater, H. A. Active Radiative Thermal Switching with Graphene Plasmon Resonators. ACS Nano 2018, 
(51) Messina, R.; Ben-Abdallah, P. Graphene-based photovoltaic cells for near-field thermal energy conversion. Sci. Rep. 2013, 3, 1383.

(52) Yang, J.; Du, W.; Su, Y.; Fu, Y.; Gong, S.; He, S.; Ma, Y. Observing of the superPlanckian near-field thermal radiation between graphene sheets. Nat. Commun. 2018, 9, 4033 .

(53) van Zwol, P. J.; Thiele, S.; Berger, C.; de Heer, W. A.; Chevrier, J. Nanoscale Radiative Heat Flow due to Surface Plasmons in Graphene and Doped Silicon. Phys. Rev. Lett. 2012, 109, 264301.

(54) Papadakis, G. T.; Zhao, B.; Buddhiraju, S.; Fan, S. Gate-Tunable Near-Field Heat Transfer. ACS Photonics 2019, 6, 709-719.

(55) Rytov, S. M. Theory of electric fluctuations and thermal radiation. Air Force Cambrige Research Center, Bedford, MA 1953,

(56) Polder, D.; Van Hove, M. Theory of Radiative Heat Transfer between Closely Spaced Bodies. Phys. Rev. B 1971, 4, 3303-3314.

(57) Rytov, S. M.; Kravtsov, Y. A.; Tatarskii, V. I. Principles of Statistical Radiophysics; 1987; Vol. 2.

(58) Brar, V. W.; Jang, M. S.; Sherrott, M.; Kim, S.; Lopez, J. J.; Kim, L. B.; Choi, M.; Atwater, H. Hybrid Surface-Phonon-Plasmon Polariton Modes in Graphene/Monolayer h-BN Heterostructures. Nano Lett. 2014, 14, 3876-3880.

(59) Dai, S.; Ma, Q.; Liu, M. K.; Andersen, T.; Fei, Z.; Goldflam, M. D.; Wagner, M.; Watanabe, K.; Taniguchi, T.; Thiemens, M.; Keilmann, F.; Janssen, G. C. a. M.; Zhu, S.-E.; Jarillo-Herrero, P.; Fogler, M. M.; Basov, D. Graphene on hexagonal boron nitride as a tunable hyperbolic metamaterial. Nat. Nanotechnol. 2015, 10, 682-686. 
(60) Kitamura, R.; Pilon, L.; Jonasz, M. Optical constants of silica glass from extreme ultraviolet to far infrared at near room temperature. Appl. Opt. 2007, 46, 8118-8133.

(61) Biehs, S.-A.; Rousseau, E.; Greffet, J.-J. Mesoscopic Description of Radiative Heat Transfer at the Nanoscale. Phys. Rev. Lett. 2010, 105, 234301.

(62) Falkovsky, L. A. Optical properties of graphene. J. Phys.: Conf. Ser. 2008, 129, 012004.

(63) Lyon, T.; Sichau, J.; Dorn, A.; Centeno, A.; Pesquera, A.; Zurutuza, A.; Blick, R. Probing Electron Spin Resonance in Monolayer Graphene. Phys. Rev. Lett. 2017, 119, 066802 . 


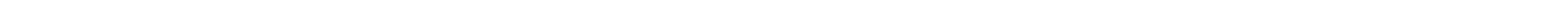




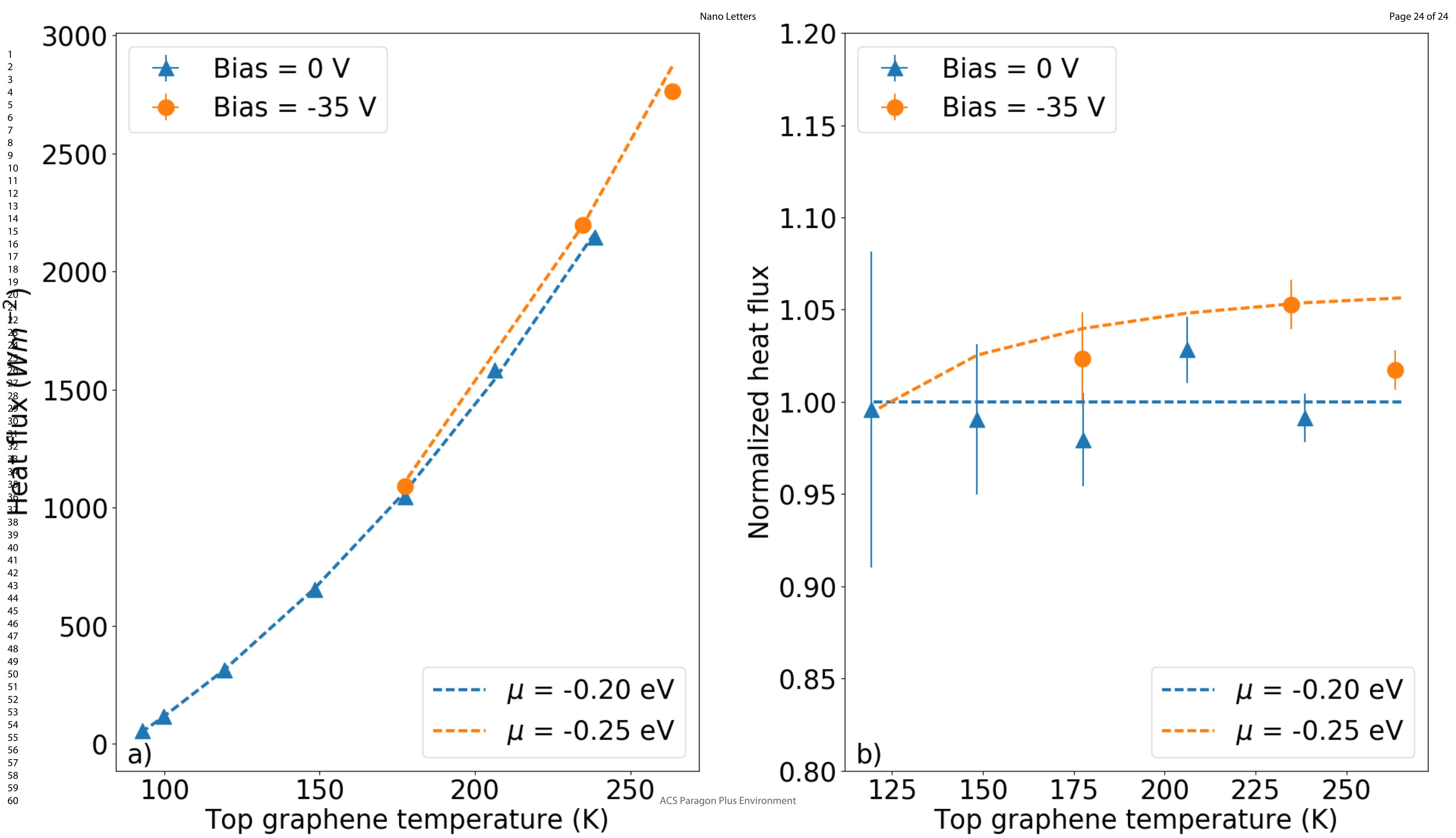

